\title{
HIGH-ENERGY RESEARCH AT CERN
}

T HE monthly, CERN Courier, which was temporarily suspended during 1961, made a welcome comeback at the beginning of 1962 . The January issue $(2$, No. $1 ; 1962)$ contains, in addition to a message from Prof. V. F. Weisskopf, the director-general of the European Organization for Nuclear Research (CERN) at Meyrin near Geneva, a comprohensive article by $J$. Baarli, the head of the health physics division, on radiation safety at CERN. Some 800 people out of a total personnel of 1,440 are subject to permanent radiation control. They are those who work either directly with radiation or in areas where a radiation dose-rate exceeding normal background has been measured. They carry $\gamma$-film badges and in the case of about 150, neutron film badges in addition. The records for 1960 show that the maximum radiation exposure for more than 700 of the 800 controlled personnel was less than $1 \mathrm{rem}$ and only one person had an exposure exceeding 5 rems. During 1961 there was a somewhat greater exposure, especially of those groups who carried out maintenance work and repair on the synchro-cyclotron accelerator, but it was still below the recommended maximum permissible radiation exposure. Other articles in the January issue include reports on the 1961 international conference on theoretical aspects of very high-energy phenomena held at CERN during Juno 5-9, 1961, and on the twelfth Solvay Physies Meeting held at the Free University of Brussels during October 9-13, 1961.

The first part of an article based on a paper prepared by the director-general for the twentieth session of the CERN council meeting on December 19, 1961, entitled "CERN and High-Energy Physics" is the main feature of the February issue of the Courier. Prof. Weisskopf, after outlining the present state of high-energy physics, considers the activities of the different high-energy research centres, particularly those contributions which have opened new avenues of research or revealed decisive results.

The main aim of high-energy physics is the study of the properties of elementary particles, and large high-energy installations are required because the smaller the object, the higher is the energy needed to penetrate into its structure. Studies to date have not yet given satisfactory answers as to the structure of the particles, but they have revealed an unexpected wealth of phenomena-nucleon structure, strange particles, weak interactions, and anti-matter-which represent a now aspect of nature, very different from experiences at lower energies.

The work with the synchro-cyclotron at CERN has been directed mainly towards weak-interaction physies, and the first important contribution was the discovery of the electron decay of the pion in 1958. Following the addition of a muon channel, the first measurement of the anomalous magnetic moment of the muon was made, and at present measurements of muon capture by hydrogen are being carried out. The proton synchrotron, which came into use in 1960, has been used for the study of peripheral collisions, and a number of new phenomena relevant to the understanding of the pion-cloud structure has been demonstrated. Experiments planned for the immediate future include the neutrino experiment, in which a neutrino beam of high energy is obtained when fast pions produced in a target decay in flight, and runs with the $81-\mathrm{cm}$. bubble chamber in a separated anti-proton beam of more than $3 \mathrm{GeV}$., in order to obtain pictures of annihilations of fast anti-protons and their by-products.

S. Weintrout

\section{DAYTIME SKY RADIANCE FROM FORTY TO EIGHTY THOUSAND FEET}

\author{
By GORDON A. NEWKIRK and JOHN A. EDDY* \\ Department of Astro-Geophysics, High Altitude \\ Observatory, Boulder, Colorado
}

\begin{abstract}
$\mathrm{T}$ HE possibility of flying large astronomical equip. ment on balloons to altitudes of 80,000-100,000 $\mathrm{ft}$. has led to increased interest in the radiance of the sky at these altitudes. The daytime sky radiance is of interest to the instrument designer, who is concerned with the contrast between the target (for example, a planet, artificial satellite, the solar corona, etc.) and the sky. It is of equal interest to the astronomer, who can learn about the sedimentation of micrometeorites through the upper atmosphere by means of the scattering of sunlight from meteoric debris, and to the meteorologist.

As part of a programme to investigate the feasibility of coronal observations from balloons, the

* Present address : National Bureau of Standards, Boulder, Colorado.
\end{abstract}

High Altitude Observatory has flown an externally occulting coronagraph up to heights of $82,000 \mathrm{ft}$. to measure the angular and the wave-length distribution of the daylight sky. The coronagraph was a modification of the instrument designed by Evans ${ }^{1}$ and used by one of us $^{2}$ for ground-based observations of the solar aureole. The balloon-borne instrument was pointed at the centre of the solar disk to within an accuracy of $\pm 1 \mathrm{~min}$. of are by the guiding gimbals developed and used in Project Stratoscope ${ }^{3-6}$.

The sky radiances were recorded in three ways: (1) photographically, at scattering angles $\varphi=9 \cdot 6^{\circ}$, $20.7^{\circ}, 31 \cdot 4^{\circ}, 40.6^{\circ}$, and $57.8^{\circ}$ at $\lambda=0.44 \mu$ with electric vector vibrating perpendicularly to the scattering plane; (2) photographically, at scattering 